\title{
Ai Consulting Healthcare Chatbot System Using Pattern Matching
}

\author{
G. Nivedhitha, E. Punarselvam, K. R. Aaghash, M. Elayabarathi, K. Rahul, R. Santhosh
}

Department of Information Technology, Muthayammal Engineering College (Autonomous), Tamilnadu, India

\author{
Article Info \\ Volume 8, Issue 3 \\ Page Number : 18-22
}

\section{Publication Issue}

May-June-2021

\section{Article History}

Accepted : 25 May 2021

Published : 30 May 2021

\section{ABSTRACT}

In today's world there are millions of diseases with various symptoms foreach, no human can possibly know about all of these diseases and the treatments associated with them. So, the problem is that there isn't any place where anyone can have the details of the diseases or the medicines/treatments. What if there is a place where you can find your health problem just by entering symptoms or the current condition of the person. It will help us to deduce the problem and to verify the solution. The proposed idea is to create a system with artificial intelligence that can meet these requirements. The AI can classify the diseases based on the symptoms and give the list of available treatments. The System is a text-to-text diagnosis chatbot that will engage patients in conversation with their medical issues and provides a personalized diagnosis based on their symptoms and profile. Hence the people can have an idea about their health and can take the right action.

Keywords : Chatbot, Pattern matching, K-Nearest Neighbour (KNN), Natural Language Processing (NLP).

\section{INTRODUCTION}

Artificial intelligence (AI), the ability of digital computer or computer-controlled robot to perform tasks commonly associated with intelligent beings. The term is frequently applied to the project of developing systems endowed with the intellectual processes characteristic of humans, such as the ability to reason, discover meaning, generalize, or learn from past experience. Since the development of the digital computer in the 1940s, it has been demonstrated that computers can be programmed to carry out very complex tasks, for example, discovering proofs for mathematical theorems or playing chess with great proficiency. Still, despite continuing advances in computer processing speed and memory capacity, there are as yet no programs that can match human flexibility over wider domains or in tasks requiring much everyday knowledge. On the other hand, some programs have attained the performance levels of human experts and professionals in performing certain specific tasks, so that artificial intelligence in this limited sense is found in applications as diverse as medical diagnosis, computer search engines, and voice or handwriting recognition. Knowledge reasoning, planning, machine learning, natural language processing, 
computer vision, robotics are the major goals of artificial intelligence.

\section{PROPOSED SYSTEM}

The proposed solution to tackle this problem, is to develop a chat bot, that will take in the symptoms from the user, uses machine learning, to come up with a classification of which type of fever it is, provide the user with the appropriate details of what he/she is suffering from, and will give them the appropriate actions to be taken. Various surveys in this area have proved that that chatbot can provide healthcare in low costs and improved treatment if the doctors. System helps users to submit their complaints and queries regarding the health. Bot can facilitate to get the common health related question and prediction of disease without a human interference.

\section{ALGORITHM}

\section{Pattern Matching}

Pattern Matching is the act of checking a given sequence of tokens for the presence of the constituents of some pattern. The match usually has to be exact: "either it will or will not be a match". Pattern matching is used to determine whether source files of high-level languages are syntactically correct. It is also used to find and replace a matching pattern in a text or code with another text/code. Any application that supports search functionality uses pattern matching in one way or another.

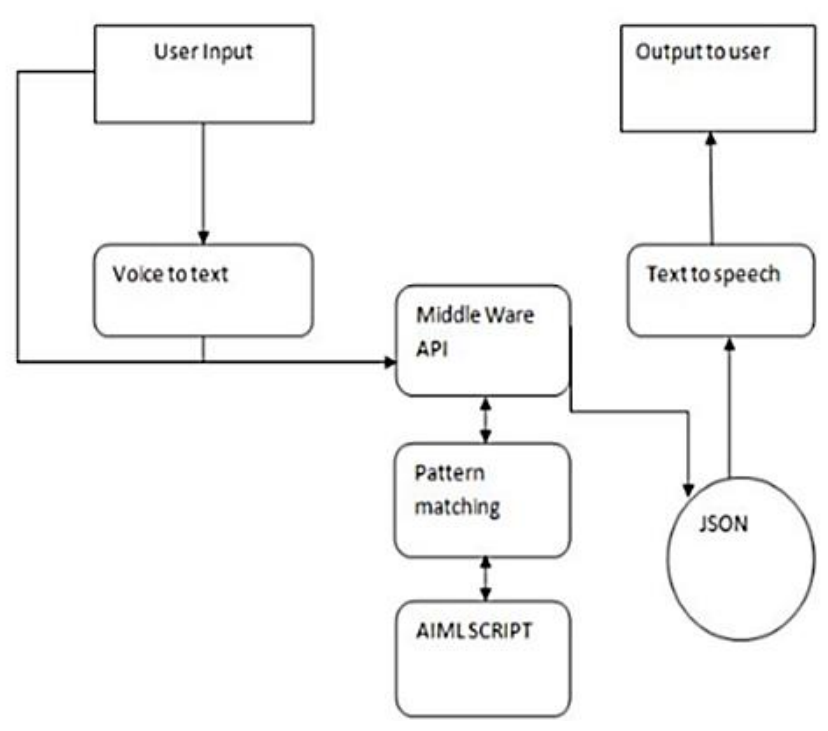

Fig 1. System Architecture

\section{ADVANTAGES}

1. User easily get healthcare information from application

2. It reduces time for users

3. Easy to implement and learn

4. User- friendliness and simplicity of the system of dialogue

5. Computer readable representation

6. There are many practical and scalable implementations available.

\section{MODULES DESCRIPTION}

\section{A. User Register}

This is the first user interface of this system, using this module user can register their details for the purpose of authentication. Once they register using this module, can easily enter into the system. Registration is one of the primary modules in any data management system. A patient's medical record management starts with registering a patient with the system It is extremely important to keep the registration module generalized in a way where it can be configured to take registration information about a patient according to the needs of the implementer. 


\section{B. User Login}

This is the second user interface of this system, the main purpose of this module to identify the user who access this system and also authenticate the user. The Login Module is a portal module that allows all users to type a user name and password to log name and can add this module on any module tab to allow users to $\log$ in to the System.

\section{User Input Voice}

After the successful authentication, user can interact with the assistant through query; User can give voice input to the assistant. The module is most popular in nowadays.

\section{Voice to Text Conversion}

The module to get the voice input from the user and convert into text format, for matching with the database and user can't retrieve the data from database through voice only. A speech recognizer is a speech engine that converts speech to text.

\section{E. Text to Voice Conversion}

This module is used to convert the output of which they retrieve from the database such as text. This Text to voice conversion mainly used for users to give the output as voice. It supports speech synthesis which means the process of generating spoken the language by machine on the basis of written input.

\section{F. User Output Voice}

This is the final user interface of this system; this module is result of the assistant (Chabot) and also deliver the output through voice. This is especially for the user purpose. This easy way of getting result, this kind of result is mainly useful for the blind person, who cannot read the out from the user interface.

\section{IMPLEMETATION}

System implementation is the important stage of project when the theoretical design is tuned into practical system. The main stages in the implementation are as follows: Planning, Training,
System testing and Changeover Planning. Planning is the first task in the system implementation. Planning means deciding on the method and the time scale to be adopted. At the time of implementation of any system people from different departments and system analysis involve. They are confirmed to practical problem of controlling various activities of people outside their own data processing departments.

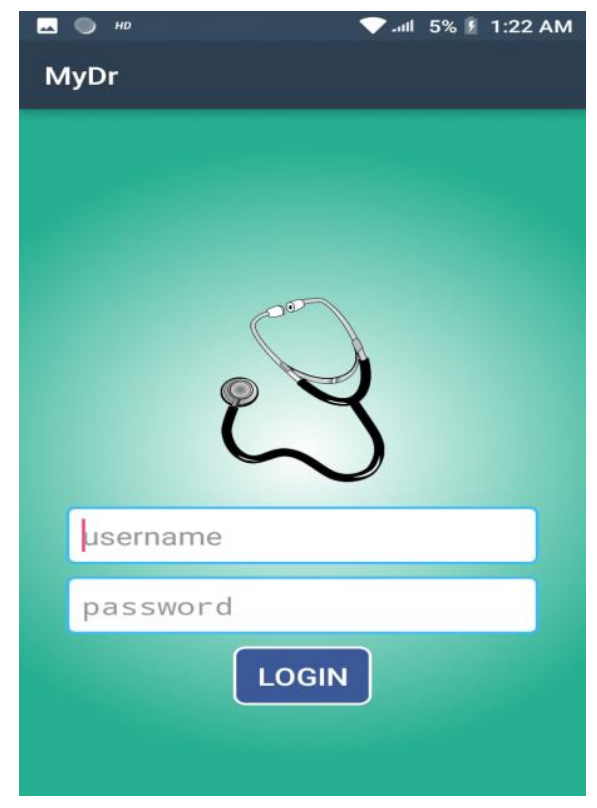

Fig.2: LOGIN PAGE

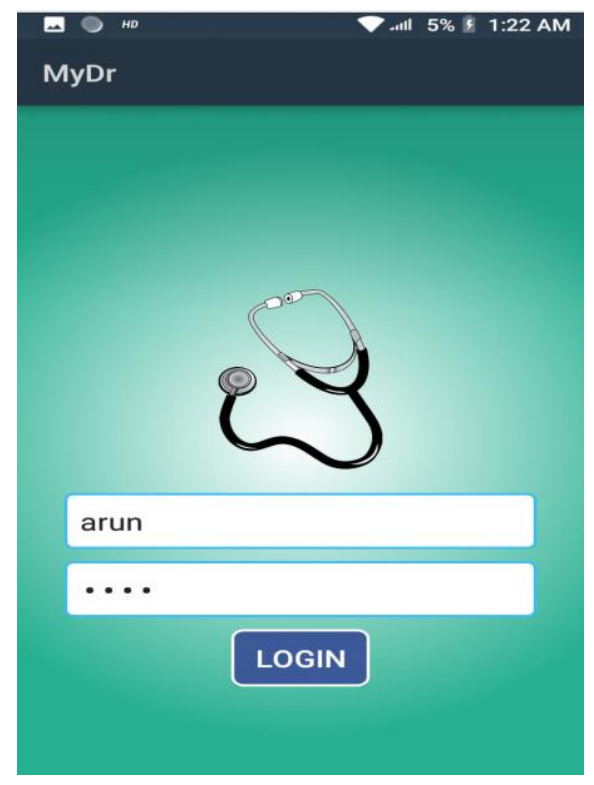

Fig.3: LOGIN DETAILS 


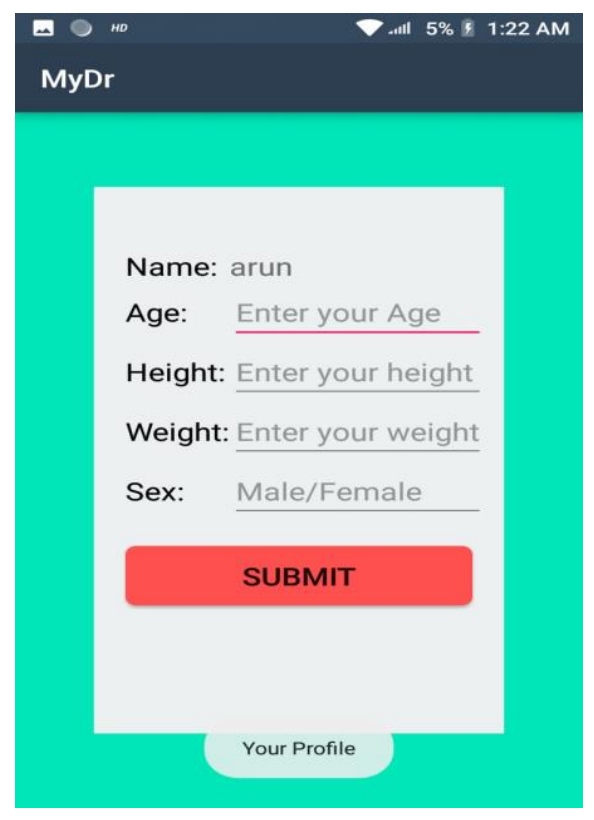

Fig 4: PROFILE PAGE

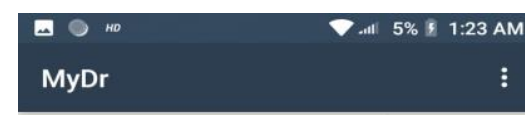

SPEAK NOW!

\section{$\stackrel{1}{P}$}

Fig 5: VOICE TO TEXT

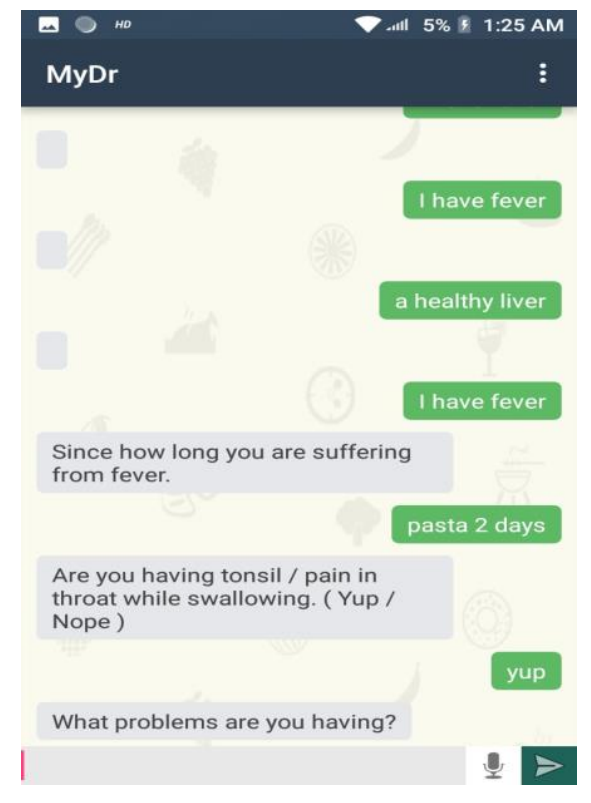

Fig 6: CHAT WITH CHATBOT

\begin{tabular}{ll}
\hline но & \\
MyDr & \\
\hline Date: & 22/aug/2019 \\
IIIness: & headache \\
Prescription: & crocine \\
\hline Date: & 23/aug/2019 AM \\
IIIness: & headache \\
Prescription: & zupar \\
\hline Date: & 24/aug/2019 \\
IIIness: & fever \\
Prescription: & calpol \\
Date: & $25 /$ aug/2019 \\
IIIness: & fever \\
Prescription: & metacine
\end{tabular}

User History

Fig 7: USER HISTORY

VII. CONCLUSION

Health information is the most important thing in the day-to-day life but most of the peoples are dose not care about that and they are affected by some other disease. So, this project has implemented the concept of health care chatbot system to the people to interact with that to know about the symptoms of the disease 
and what are the precaution for their health and also the hospital details for the users. Proposed worked the chat bot can interact with people through both text and voice conversation It is very easy to use for all the users and get the information about the disease details quickly. This part of the system can help the users to known about the depth of the disease details attack and how they can precaution from that.

\section{FUTURE ENHANCEMENT}

The role played by chatbot can sometimes be beyond the scope and user may require consulting a doctor for taking health related tests. In such situations, chatbot can be helpful if it can be made to set up an appointment with an efficient doctor based on their schedule. Also, it will be beneficial if the symptoms and disease identified by the chatbot can be made into a report and automatically forwarded to an available doctor where he can further assist the user with more advices and future measures to maintain their health. A video call with a specialized doctor can also be made depending on the availability of the user rather than based on the availability of doctors.

\section{REFERENCES}

[1]. Sameera A. Abdul-Kader, Dr. John Woods. \&quot;Survey on Chatbot Design Techniques in Speech Conversation Systems, School of Computer Science and Electronic Engineering/University of Essex Colchester/ UK\&quot; 2019.

[2]. S. Divya, V. Indumathi, S. Ishwarya, M. Priyasankari, S. Kalpana Devi, \&quot;A SelfDiagnosis Medical Chatbot Using Artificial Intelligence\&quot; J. Web Dev. Web Des., vol. 3, no. 1, pp. 1-7, 2018.

[3]. Mrs. RashmiDharwadkar, Dr.Mrs. Neeta A. Deshpande \&quot;A Medical ChatBot\&quot;" in International Journal of Computer Trends and Technology (IJCTT) V60(1):41-45, June 2018.

[4]. DivyaMadhu,Neeraj Jain C. J, ElmySebastain, ShinoyShaji, AnandhuAjayakumar. A Novel Approach for Medical Assistance Using Trained Chatbot, International Conference on Inventive
Communication and Computational Technologies (ICICCT 2017).

[5]. R. Ranoliya, N. Raghuwanshi, S. Singh, \&quot;Chatbot for university related FAQs\&quot; 2017 Int. Conf. Adv. Comput. Commun. Informatics ICACCI 2017, pp. 1525-1530, September 2017.

[6]. Amiya Kumar Tripathy ,RebeckCarvalho , KeshavPawaskar , SurajYadav, "Mobile based healthcare management using artificial intelligence" in International Conference on Technologies for Sustainable Development (ICTSD), 2015, 4-6 Feb, 2015.

[7]. Kuligowska, Karolina. (2015). Commercial Chatbot: Performance Evaluation, Usability Metrics and Quality Standards of Embodied Conversational Agents. Professionals Center for Business Research. 2. 1-16.10.18483/PCBR.22.

[8]. ShwetaTaneja ,Charu Gupta , KratikaGoyal , DharnaGureja, “An Enhanced K-Nearest Neighbor Algorithm Using Information Gain and Clustering”, 2014 Fourth International Conference on Advanced Computing Communication Technologies. doi:10.1109/acct.2014.22

[9]. Pu Han, Si Shen, Dongbo Wang, Yanyun Liu, “The influence of word normalization in English document clustering", 2012 IEEE International Conference on Computer Science and Automation Engineering (CSAE). doi:10.1109/csae.2012.6272740

[10]. S. du Preez, M. Lall, S. Sinha, \&quot;An intelligent web-based voice chat bot\&quot; EUROCON 2009 EUROCON\&\#39;09.IEEE, pp. 386-391, 2009.

\section{Cite this article as :}

G. Nivedhitha, E. Punarselvam, K. R. Aaghash, M. Elayabarathi, K. Rahul, R. Santhosh, "Ai Consulting Healthcare Chatbot System Using Pattern Matching", International Journal of Scientific Research in Science and Technology (IJSRST), Online ISSN : 2395-602X, Print ISSN : 2395-6011, Volume 8 Issue 3, pp. 18-22, May-June 2021. Available at doi : https://doi.org/10.32628/IJSRST2182112 Journal URL : https://ijsrst.com/IJSRST2182112 